\title{
Highlight report: liver to bone communication
}

\author{
Hassan Ahmed ${ }^{1} \cdot$ Mohamed S. Ahmed $^{2} \cdot$ Alsagher O. Ali $^{3} \cdot$ Seddik Hammad ${ }^{4,5}(\mathbb{C}$
}

Received: 1 July 2019 / Accepted: 4 July 2019 / Published online: 9 July 2019

(c) Springer-Verlag GmbH Germany, part of Springer Nature 2019

Recently, Sabrina Ehnert and colleagues from Tuebingen University (Germany) published a comprehensive review about hepatic osteodystrophy (Ehnert et al. 2019). Currently, numerous studies focus on a better understanding of liver disease (Jansen et al. 2017; Vartak et al. 2016; Nussler et al. 2014; Hammad et al. 2018) and mechanisms of hepatotoxicity (Grinberg et al. 2014, 2018; Schenk et al. 2017; Godoy et al. 2013, 2015, 2016; Hammad et al. 2017, 2018; Kodidela et al. 2017; Leist et al. 2017). However, relatively little is known how disturbed liver functions influence other organs. The majority of patients with chronic liver diseases suffer from hepatic osteodystrophy (Nakchbandi and van der Merwe 2009; Diamond et al. 1990; Wibaux et al. 2011; Tsai et al. 2013; Nussler et al. 2014). Hepatic osteodystrophy increases the risk of bone fractures which are difficult to treat and affect the long-term prognosis of patients (Angulo et al. 2011; Guañabens et al. 2010; Guarino et al. 2016; Goel and Kar 2010). In the present review, Ehnert and colleagues

Seddik Hammad

seddik.hammad@vet.svu.edu.eg;

seddik.hammad@medma.uni-heidelberg.de

Hassan Ahmed

hassan-younes@vet.svu.edu.eg

Mohamed S. Ahmed

m.s.ahmed@vet.svu.edu.eg

Alsagher O. Ali

Alsagher.ali@vet.svu.edu.eg

1 Department of Physiology, Faculty of Veterinary Medicine, South Valley University, Qena 83523, Egypt

2 Department of Poultry Diseases, Faculty of Veterinary Medicine, South Valley University, Qena 83523, Egypt

3 Department of Animal Medicine, Faculty of Veterinary Medicine, South Valley University, Qena 83523, Egypt

4 Department of Forensic Medicine and Veterinary Toxicology, Faculty of Veterinary Medicine, South Valley University, Qena 83523, Egypt

5 Molecular Hepatology Section, Department of Medicine II, Medical Faculty Mannheim, Heidelberg University, 68167 Mannheim, Germany summarize and discuss current knowledge of the molecular mechanisms responsible for hepatic osteodystrophy:

- Compromised vitamin D metabolism.

- Alterations in transforming growth factor beta (TGF- $\beta$ )induced effects.

- Compromised bone morphogenetic protein (BMP) signaling.

- Altered expression of histone deacetylases (HDACs).

- Functional loss mutations of sclerostin.

Based on these mechanisms, the authors discuss therapeutic perspectives and possibilities or early diagnosis. The article is a must-read for anyone interested in the mechanisms how liver diseases compromise bone metabolism.

\section{Compliance with ethical standards}

Conflict of interest The authors declare that they have no conflict of interest.

\section{References}

Angulo P, Grandison GA, Fong DG, Keach JC, Lindor KD, Bjornsson E, Koch A (2011) Bone disease in patients with primary sclerosing cholangitis. Gastroenterology 140(1):180-188. https://doi. org/10.1053/j.gastro.2010.10.014

Diamond T, Stiel D, Lunzer M, Wilkinson M, Roche J, Posen S (1990) Osteoporosis and skeletal fractures in chronic liver disease. Gut 31(1):82-87

Ehnert S, Aspera-Werz RH, Ruoß M, Dooley S, Hengstler JG, Nadalin S, Relja B, Badke A, Nussler AK (2019) Hepatic osteodystrophy-molecular mechanisms proposed to favor its development. Int J Mol Sci 20(10):pii: E2555. https://doi.org/10.3390/ijms2 0102555 (review)

Godoy P, Hewitt NJ, Albrecht U, Andersen ME, Ansari N, Bhattacharya S, Bode JG, Bolleyn J, Borner C, Böttger J, Braeuning A, Budinsky RA, Burkhardt B, Cameron NR, Camussi G, Cho CS, Choi YJ, Craig Rowlands J, Dahmen U, Damm G, Dirsch O, Donato MT, Dong J, Dooley S, Drasdo D, Eakins R, Ferreira KS, Fonsato V, Fraczek J, Gebhardt R, Gibson A, Glanemann 
M, Goldring CE, Gómez-Lechón MJ, Groothuis GM, Gustavsson L, Guyot C, Hallifax D, Hammad S, Hayward A, Häussinger D, Hellerbrand C, Hewitt P, Hoehme S, Holzhütter HG, Houston JB, Hrach J, Ito K, Jaeschke H, Keitel V, Kelm JM, Kevin Park B, Kordes C, Kullak-Ublick GA, LeCluyse EL, Lu P, LuebkeWheeler J, Lutz A, Maltman DJ, Matz-Soja M, McMullen P, Merfort I, Messner S, Meyer C, Mwinyi J, Naisbitt DJ, Nussler AK, Olinga P, Pampaloni F, Pi J, Pluta L, Przyborski SA, Ramachandran A, Rogiers V, Rowe C, Schelcher C, Schmich K, Schwarz M, Singh B, Stelzer EH, Stieger B, Stöber R, Sugiyama Y, Tetta C, Thasler WE, Vanhaecke T, Vinken M, Weiss TS, Widera A, Woods CG, Xu JJ, Yarborough KM, Hengstler JG (2013) Recent advances in $2 \mathrm{D}$ and $3 \mathrm{D}$ in vitro systems using primary hepatocytes, alternative hepatocyte sources and non-parenchymal liver cells and their use in investigating mechanisms of hepatotoxicity, cell signaling and ADME. Arch Toxicol 87(8):1315-1530. https ://doi.org/10.1007/s00204-013-1078-5

Godoy P, Schmidt-Heck W, Natarajan K, Lucendo-Villarin B, Szkolnicka D, Asplund A, Björquist P, Widera A, Stöber R, Campos G, Hammad S, Sachinidis A, Chaudhari U, Damm G, Weiss TS, Nüssler A, Synnergren J, Edlund K, Küppers-Munther B, Hay DC, Hengstler JG (2015) Gene networks and transcription factor motifs defining the differentiation of stem cells into hepatocyte-like cells. J Hepatol 63(4):934-942. https://doi. org/10.1016/j.jhep.2015.05.013 (Erratum. In: J Hepatol. 2016 Feb; 64(2):525-6)

Godoy P, Widera A, Schmidt-Heck W, Campos G, Meyer C, Cadenas C, Reif R, Stöber R, Hammad S, Pütter L, Gianmoena K, Marchan R, Ghallab A, Edlund K, Nüssler A, Thasler WE, Damm G, Seehofer D, Weiss TS, Dirsch O, Dahmen U, Gebhardt R, Chaudhari U, Meganathan K, Sachinidis A, Kelm J, Hofmann U, Zahedi RP, Guthke R, Blüthgen N, Dooley S, Hengstler JG (2016) Gene network activity in cultivated primary hepatocytes is highly similar to diseased mammalian liver tissue. Arch Toxicol 90(10):2513-2529. https://doi.org/10.1007/s00204-016-1761-4

Goel V, Kar P (2010) Hepatic osteodystrophy. Trop Gastroenterol $31(2): 82-86$ (review)

Grinberg M, Stöber RM, Edlund K, Rempel E, Godoy P, Reif R, Widera A, Madjar K, Schmidt-Heck W, Marchan R, Sachinidis A, Spitkovsky D, Hescheler J, Carmo H, Arbo MD, van de Water B, Wink S, Vinken M, Rogiers V, Escher S, Hardy B, Mitic D, Myatt G, Waldmann T, Mardinoglu A, Damm G, Seehofer D, Nüssler A, Weiss TS, Oberemm A, Lampen A, Schaap MM, Luijten M, van Steeg H, Thasler WE, Kleinjans JC, Stierum RH, Leist M, Rahnenführer J, Hengstler JG (2014) Toxicogenomics directory of chemically exposed human hepatocytes. Arch Toxicol 88(12):2261-2287. https://doi.org/10.1007/s00204-014-1400-x

Grinberg M, Stöber RM, Albrecht W, Edlund K, Schug M, Godoy P, Cadenas C, Marchan R, Lampen A, Braeuning A, Buhrke T, Leist M, Oberemm A, Hellwig B, Kamp H, Gardner I, Escher S, Taboureau O, Aguayo-Orozco A, Sachinidis A, Ellinger-Ziegelbauer H, Rahnenführer J, Hengstler JG (2018) Toxicogenomics directory of rat hepatotoxicants in vivo and in cultivated hepatocytes. Arch Toxicol 92(12):3517-3533. https://doi.org/10.1007/s0020 4-018-2352-3

Guañabens N, Cerdá D, Monegal A, Pons F, Caballería L, Peris P, Parés A (2010) Low bone mass and severity of cholestasis affect fracture risk in patients with primary biliary cirrhosis. Gastroenterology 138(7):2348-2356. https://doi.org/10.1053/j.gastr o.2010.02.016

Guarino M, Loperto I, Camera S, Cossiga V, Di Somma C, Colao A, Caporaso N, Morisco F (2016) Osteoporosis across chronic liver disease. Osteoporos Int 27(6):1967-1977. https://doi.org/10.1007/ s00198-016-3512-z (review)

Hammad S, Braeuning A, Meyer C, Mohamed FEZA, Hengstler JG, Dooley S (2017) A frequent misinterpretation in current research on liver fibrosis: the vessel in the center of CCl4-induced pseudolobules is a portal vein. Arch Toxicol 91(11):3689-3692. https ://doi.org/10.1007/s00204-017-2040-8

Hammad S, Othman A, Meyer C, Telfah A, Lambert J, Dewidar B, Werle J, Nwosu ZC, Mahli A, Dormann C, Gao Y, Gould K, Han M, Yuan X, Gogiashvili M, Hergenröder R, Hellerbrand C, Thomas M, Ebert MP, Amasheh S, Hengstler JG, Dooley S (2018) Confounding influence of tamoxifen in mouse models of Cre recombinase-induced gene activity or modulation. Arch Toxicol 92(8):2549-2561. https://doi.org/10.1007/s00204-018-2254-4

Jansen PL, Ghallab A, Vartak N, Reif R, Schaap FG, Hampe J, Hengstler JG (2017) The ascending pathophysiology of cholestatic liver disease. Hepatology 65(2):722-738. https://doi.org/10.1002/ hep.28965 (review)

Kodidela S, Govatati S, Matha SK, Korla SV, Naidu BP, Thippannagari L, Bramhachari PV, Bhanoori M, Nallanchakravarthula V (2017) Impact of alcohol on hepatic mitochondrial DNA damage in streptozotocin diabetic rats. JEAAS 2(2):90-101. https://doi. org/10.20454/jeaas.2017.1225

Leist M, Ghallab A, Graepel R, Marchan R, Hassan R, Bennekou SH, Limonciel A, Vinken M, Schildknecht S, Waldmann T, Danen E, van Ravenzwaay B, Kamp H, Gardner I, Godoy P, Bois FY, Braeuning A, Reif R, Oesch F, Drasdo D, Höhme S, Schwarz M, Hartung T, Braunbeck T, Beltman J, Vrieling H, Sanz F, Forsby A, Gadaleta D, Fisher C, Kelm J, Fluri D, Ecker G, Zdrazil B, Terron A, Jennings P, van der Burg B, Dooley S, Meijer AH, Willighagen E, Martens M, Evelo C, Mombelli E, Taboureau O, Mantovani A, Hardy B, Koch B, Escher S, van Thriel C, Cadenas C, Kroese D, van de Water B, Hengstler JG (2017) Adverse outcome pathways: opportunities, limitations and open questions. Arch Toxicol 91(11):3477-3505. https://doi.org/10.1007/s0020 4-017-2045-3 (review)

Nakchbandi IA, van der Merwe SW (2009) Current understanding of osteoporosis associated with liver disease. Nat Rev Gastroenterol Hepatol 6(11):660-670. https://doi.org/10.1038/nrgastro.2009.166 (review)

Nussler AK, Wildemann B, Freude T, Litzka C, Soldo P, Friess H, Hammad S, Hengstler JG, Braun KF, Trak-Smayra V, Godoy P, Ehnert S (2014) Chronic $\mathrm{CCl}_{4}$ intoxication causes liver and bone damage similar to the human pathology of hepatic osteodystrophy: a mouse model to analyse the liver-bone axis. Arch Toxicol 88(4):997-1006. https://doi.org/10.1007/s00204-013-1191-5

Schenk A, Ghallab A, Hofmann U, Hassan R, Schwarz M, Schuppert A, Schwen LO, Braeuning A, Teutonico D, Hengstler JG, Kuepfer L (2017) Physiologically-based modelling in mice suggests an aggravated loss of clearance capacity after toxic liver damage. Sci Rep 7(1):6224. https://doi.org/10.1038/s41598-017-04574-Z

Tsai CF, Liu CJ, Chen TJ, Chu CJ, Lin HC, Lee FY, Su TP, Lu CL (2013) Increased incidence of orthopedic fractures in cirrhotic patients: a nationwide population-based study. J Hepatol 58(4):706-714. https://doi.org/10.1016/j.jhep.2012.12.001

Vartak N, Damle-Vartak A, Richter B, Dirsch O, Dahmen U, Hammad S, Hengstler JG (2016) Cholestasis-induced adaptive remodeling of interlobular bile ducts. Hepatology 63(3):951-964. https://doi. org/10.1002/hep. 28373

Wibaux C, Legroux-Gerot I, Dharancy S, Boleslawski E, Declerck N, Canva V, Mathurin P, Pruvot FR, Cortet B (2011) Assessing bone status in patients awaiting liver transplantation. Joint Bone Spine 78(4):387-391. https://doi.org/10.1016/j.jbspin.2011.03.001

Publisher's Note Springer Nature remains neutral with regard to jurisdictional claims in published maps and institutional affiliations. 